\title{
The Establishment of Counterpart Selection Metrics for Supply Chain Finance Execution
}

\author{
Rui ZHANG \\ Faculty of management Wuhan University of Technology Wuhan, P. R. China \\ Ix_zr@163.com
}

Keywords: Counterpart selection metrics, Supply chain finance.

\begin{abstract}
This paper introduces the approaches for the establishment of counterpart selection metrics for supply chain finance execution. With frequency statistics and empirical analysis method, we raised 18 counterparts selection and evaluation metrics for supply chain finance execution, besides, it is established counterpart selection and evaluation model for supply chain finance execution.
\end{abstract}

\section{Introduction}

The counterpart selection is one of the most critical parts for core enterprise operation under supply chain financing model. It can be divided into two parts to discuss about the relationship between core enterprises and their counterparts under supply chain financing model. On one hand, core enterprises play a dominate role, which invest sufficient money and talents on the operation to help the counterparts, one the other hand, the implementation of supply chain financing will have risk, but the excellent counterparts will eliminate this kind of risks to some extent.

\section{The establishment of counterpart selection metrics for supply chain execution}

(1)The principle of metrics selection

Avoid indicators duplicate; different indicators explain the counterpart condition in different way, which will lead to overlapping or duplication; core enterprise perspective is the emphasis of this investigation. How to eliminate the risk and maximize their own benefit for those core enterprises in the supply chain finance will be the focus need be investigated. When designing this metric system, we take counterpart's own growing into the consideration, meanwhile, if counterpart could fulfill the further development of core enterprise was regarded as an important question need be discussed, and the selection of counterpart is a key issue because his experience and capability will play a decisive role on enterprise management level. Therefore, when determining counterpart performance metrics, we take enterprise manager's information as an important aspect to evaluate the enterprise overall performance.

(2) The source of metrics selection

The source metrics come from existing documentation and the investigations on core enterprise, which can be generally divided into three parts, primary resource, important resource and referential resource, in order to keep the metrics align, the source date are the lowest detailed metrics we collected.

(3) Preliminary metrics selection on frequency statistics method

This paper preliminary selects the metrics from three primary resources with frequency statistics method, selection process apply for above metrics selection principle based on frequency statistics method, which eliminate low frequency indicator and finally get the preliminary metrics.

(4) Filter the metrics based on empirical analysis method

When we preliminary select evaluation metrics with frequency statistic method, the system completeness is very important, which may course evaluation content overlapping or duplicated, in order to make sure each indicator contains more information, but still precise, we established a more optimized metrics system to reduce the connection of each indicator and improve the operability of metrics system. In this point of view, based on preliminary metrics selection, we applied expert grading method to filter out counterpart selection and evolution metrics of supply 
chain finance execution, and influence factor in supply chain finance and the supply chain finance environment for small and middle sized enterprise should be fully took into the consideration.

Author sent out 30 questionnaire, received 28 questionnaire, 28 of them are valid. In each group, we summarized expert rating and calculated averaged score, and according to the score of each index, we sort out the impact factor on counterpart selection and evaluation for supply chain finance execution from big to small.

(5)Empirical analysis to determine the evaluation index

Above metrics almost included all factors influenced counterpart selection and evaluation on supply chain finance, and some of the index associated with each other which will lead to information duplication, therefore, the validity of evaluation will reduced. In this paper, we combined investigation results and expert advises to analyze 25 index correlation and containment. This paper Delete the seven indicators such as macro environment, the expected cash flows, and so on. Finally, we Get 18 specific indicators as shown in Table 1-1.

Table1-1 Counterpart selection and evaluation metrics for supply chain finance.

\begin{tabular}{|c|c|}
\hline \multirow{2}{*}{ Manager condition } & The quality of manager \\
\hline & Management capability of manager \\
\hline \multirow{2}{*}{ Debt paying ability } & Times interest earned \\
\hline & Quick ratio \\
\hline \multirow{3}{*}{ Credit condition } & Loan's performance \\
\hline & Trading performance \\
\hline & Other credit record \\
\hline \multirow{3}{*}{ R\&D ability } & New product R\&D ability \\
\hline & The intensity of R\&D investment \\
\hline & New product in sales proportion \\
\hline \multirow{3}{*}{ Market condition } & Sales growth \\
\hline & Revenue growth \\
\hline & Market growth \\
\hline \multirow{3}{*}{ Operation capability } & Account receivable turnover rate \\
\hline & Inventory turnover ratio \\
\hline & Velocity of liquid asset \\
\hline \multirow{2}{*}{ Cooperation condition } & Dependent on their product \\
\hline & Time and frequency of cooperation \\
\hline
\end{tabular}

According to above analysis, we know that select and evaluate counterpart of supply chain finance based on 7 factors: manager condition, Debt paying ability, Credit condition, R\&D ability, Market condition, Operation capability, Cooperation condition. We could establish infrastructure based on above 7 factors for counterpart selection and evaluation metrics of supply chain finance execution.

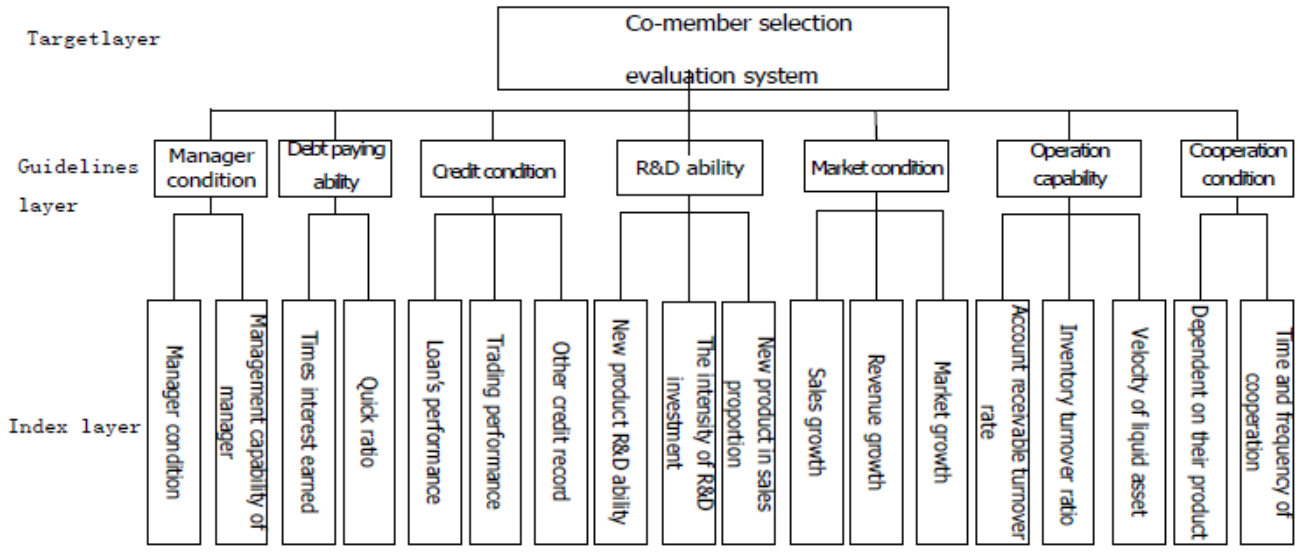

Figure1-1 Co-member selection evaluation system 


\section{Counterpart selection model for supply chain finance execution.}

If core enterprise received application letters from counterpart, firstly, please combined historical date, current project information to evaluate the aggregate risk of counterpart enterprise, secondly, according to evaluation value and evaluation standard to analyze and work out cooper able level to control the investment risk, this paper based on above approaches to established below model for supply chain finance execution.

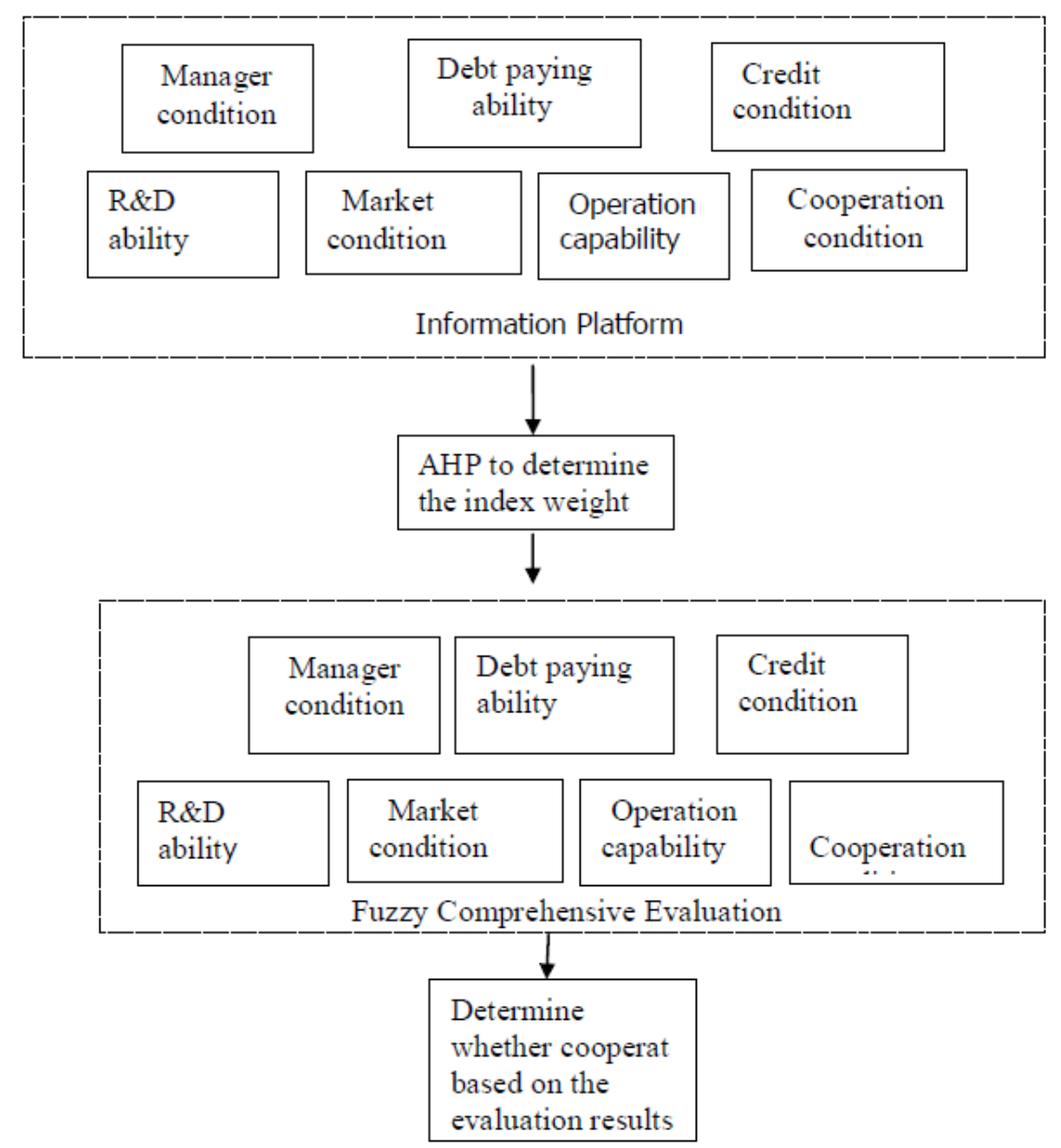

Figure2-1Counterpart selection model for supply chain finance execution

\section{Conclusion}

Core enterprise used their credit to involve in supply chain finance from the beginning to the end, it will increase the risk if any part appear issue, which may have negative impact on the whole supply chain effectiveness and efficiency in the end, therefore, excellent counterpart selection and evaluation metrics will make sure the smoothly operation for the whole supply chain, the counterpart selection and evaluation metrics and working model provided in the paper will facilitate the growth of supply chain finance.

\section{References}

[1] Hackett-REL, Research Alert: Top U.S.Companies See\$72 Billion in Improved Working Capital [J].Yet Annual Survey Identifies an Additional\$450 Billion in Potential Cash, 2006, 122132.

[2] Lewis Julian, Demand Drive Supply Chain Finance [J], Treasury perspectives, 2007, 5:46-57.

[3] Demica.Demand and Supply:Supply chain finance[J],Demica Report Seris,May, 2008,178-189. 